\title{
Wide-band Hybrid Single-Ended and Differential LNA Designs Using GaAs pHEMTs and Comparison with In-house InP pHEMT Based LNA Design
}

\author{
N. Ahmad ${ }^{\star}$, S. Arshad, B. Boudjelida, S. Boulay and M. Missous
}

School of Electrical and Electronic Engineering, University of Manchester, UK.

\begin{abstract}
This work presents single-ended and differential MIC LNA designs to meet the requirements for the Square Kilometre Array (SKA) at the low frequency band ranging from 0.3-1.0 GHz Hall (2004). A series of single-ended MIC LNAs on 50 $\Omega$ system, with various topologies have been designed to study their NF, gain, SWR, power and stability characteristics. Subsequently, differential LNAs using commercial GaAs pHEMTs were designed and optimised for the Orthogonal Ring Antenna (ORA); the proposed antennas for the phased array of the SKA. Test structures have been developed to measure the four-port differential LNAs using two-port Vector Network Analyser (VNA) and Noise Figure Analyser (NFA). The measurement techniques were presented in Abidi \& Leete (1999); Belostotski \& Haslett (2008). The above results were then compared with the low-power double stage differential to single-ended MIC LNA design Arshad et al. (2009) developed using an in-house fabricated novel InGaAs/InAlAs/InP pHEMTs Bouloukou et al. (2006).
\end{abstract}

\section{Introduction}

This work presents LNA designs for the low frequency band of SKA. The optimum goal of this work is to achieve a lowpower, low noise differential to single-ended amplifier using commercially available GaAs pHEMTs as well as in-house developed InP based pHEMTs. The first design discussed here is a single-ended MIC LNA that has been fabricated and measured. Secondly a differential to single-ended MIC LNA design using commercial GaAs pHEMTs is presented which is further compared to a similar topology LNA realised using InP based pHEMTs.

\section{Hybrid single-ended MIC LNA}

The hybrid single-ended MIC LNA was designed and optimised at frequency range of $0.5-2 \mathrm{GHz}$. The MIC LNA using GaAs transistors were fabricated and measured using inhouse facilities. The simulated gain and Noise Figure are compared with the measured data. Results show that both are in acceptable tolerance. The measured Noise Figure of $<0.7 \mathrm{~dB}$ is achieved from $0.5-1.0 \mathrm{GHz}$ as shown in Fig 1.

At the same frequency, the measured gain is in range of $15 \mathrm{~dB}$ to $20 \mathrm{~dB}$ as depicted in Fig 2. The design demonstrates stability without oscillation over entire desired frequency range. The power consumption of this LNA is $280 \mathrm{~mW}$.

\section{Differential to Single-Ended LNA Design Using Commercial GaAs pHEMTs}

In this double stage design, commercial GaAs transistors from Avago Technologies were used. Transistor ATF_54143 (E-mode pHEMT) were used as amplifiers while transistor

^ norhawati.ahmad@postgrad.manchester.ac.uk



Fig. 1: Simulated and Measured NF on single-ended single stage MIC LNA

ATF_35143 (D-mode pHEMT) were used as active loads. Both transistors have $800 \mu \mathrm{m}$ gate width.

The first stage is a Differential to Single-Ended conversion stage using self biased topology while, the second stage act as a gain plus stability boosting stage. The design is optimized for the band $0.3-1.0 \mathrm{GHz}$.

The simulation results on Noise Figure and gain response are presented in Fig 3 and Fig 4, respectively.

The figures show that NF and NFmin were both $<0.5 \mathrm{~dB}$ for the frequency band of interest. The simulations predict an unconditionally stable design consuming $650 \mathrm{~mW}$ of power. 


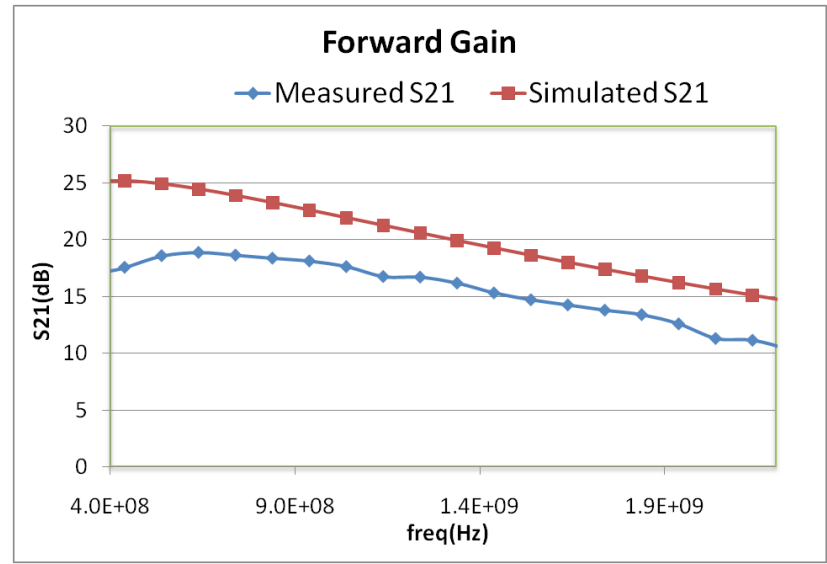

Fig. 2: Simulated and measured forward gain (S21) of single-ended single stage MIC LNA

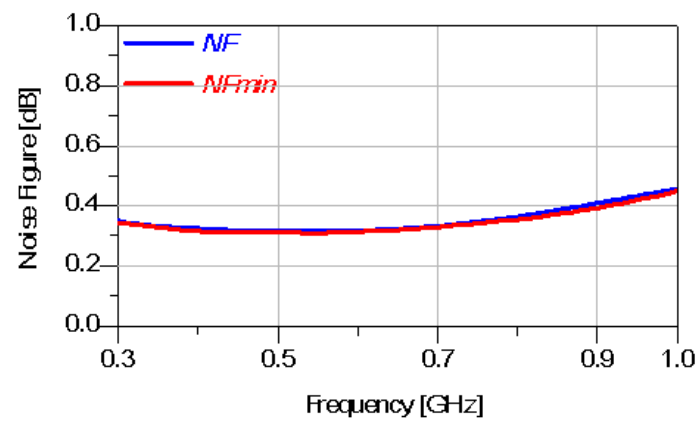

Fig. 3: Simulated NF and NFmin for differential LNA

\section{Differential To Single-Ended LNA Design Using In-House InP pHEMTs}

In this part, the LNA is designed based on in-house InP pHEMT with varying gate width. However, only $4 \times 200 \mu \mathrm{m}$ device results are presented.

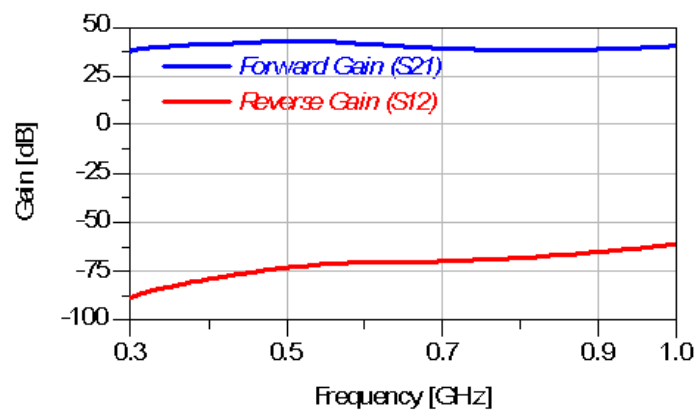

Fig. 4: Simulated forward (S21) and reverse (S12) gain of the differential LNA

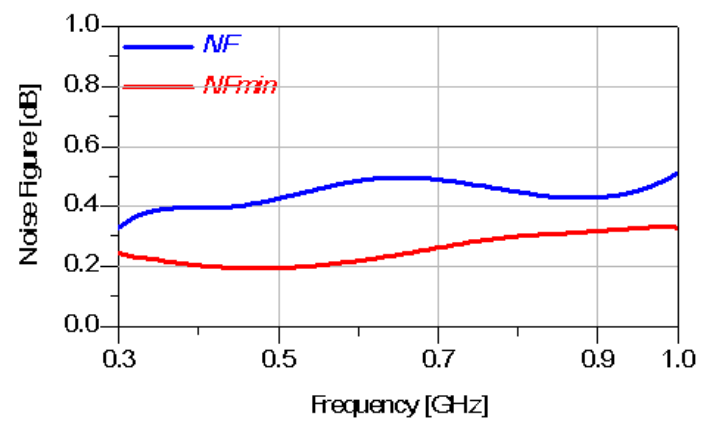

Fig. 5: Simulated NF and NFmin of in-house InP pHEMT

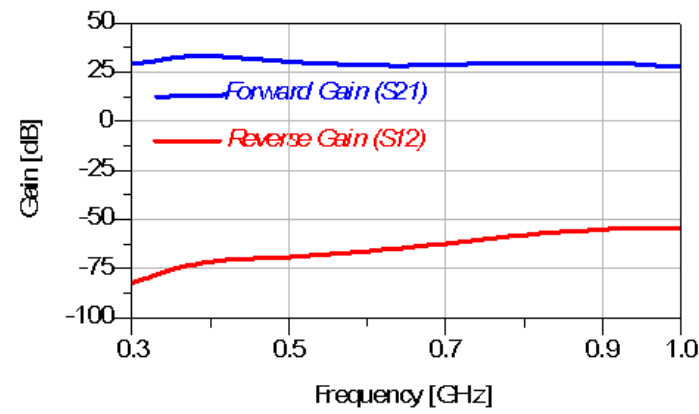

Fig. 6: Simulated forward (S21) and reverse (S12) gains of in-house InP pHEMT

For comparison purposed, the topology is similar to that of the differential LNA using Commercial GaAs pHEMTs. The designs are optimised for low noise and low power performance.

The Noise Figure shown in Fig 5 were as good as the previous LNA where both were $<0.5 \mathrm{~dB}$ over the entire frequency range considered.

The LNA gains shown in Fig 6 were comparable with the LNA using commercial GaAs pHEMTs.

In this design the total power consumption was $125 \mathrm{~mW}$ which is far less than the previous GaAs LNA design by a factor of $\sim 5$.

\section{Conclusions}

The work presented here has highlighted and demonstrated designs and measurements on differential to single-ended LNA design. The InP transistor designs have shown comparable or lower Noise Figure, NFmin and gain with superior power consumption as compared to GaAs based designs by a factor of $\sim 5$. 


\section{References}

Hall P.J. The square kilometre array: An international engineering perspective. in Square Kilometre Array Meeting (SKA). 2004. Penticton, CANADA: Springer.

Abidi A.A. and J.C. Leete, De-embedding the noise figure of differential amplifiers. Solid-State Circuits, IEEE Journal of, 1999. 34(6): p. 882-885.

Belostotski L. and J.W. Haslett, A Technique for Differential Noise Figure Measurement of Differential LNAs. Instrumentation and Measurement, IEEE Transactions on, 2008. 57(7): p. 1298-1303.

Arshad S., B. Boudjelida and M. Missous Sub-0.5 dB NF Differential-Single Ended LNA using InGaAs InAlAs InP pHEMTs for Radio Astronomy Applications. in UK Semiconductors 2009. 2009. Sheffield, UK.

Bouloukou A.S., D. Kettle, J. Sly, M. Missous. Novel High Breakdown InGaAs/InAlAs pHEMTs for radio astronomy applications. in Proceedings of the 4th ESA Workshop on Millimeter Wave Technology and Applications (7th MINT Millimiter-Wave International Symposium). 2006. Espoo, Finland. 